\title{
Dynamic Modeling and Simulation of ball bearing faults in aero-engine dual rotor-bearing-stator coupling system
}

\author{
Wei Zhang,Hao-haoHe,An-boMing*,Zheng-weiYang
}

Xi'an Research Institute of Hi-Tech, Shaanxi, P. R. China

*729814553@qq.com

Keywords: dual rotor system; ball bearing; localized fault model; numerical integral

\begin{abstract}
.
In order to conduct the thorough research to the aero-engine, aiming at dual rotor-bearing system of practical aero-engine, a new dual rotor-ball bearing-stator coupling system dynamic model is established. stator motion, the flexible support, squeeze film damper (SDF) were considered in the model, meanwhile the nonlinear factors of ball bearing such as clearance of bearing, nonlinear Hertzian contract force between balls and races, and the varying compliance vibration because of periodical variety of the support stiffness were fully considered. On this basis, established the rolling bearing inner ring damage dynamics model in the coupling system and he Runge-Kutta method was used to simulate and analyze the system, the simulation results verify the dynamic character of the coupling dynamic model with localized bearing faults and show the model is correct and effective.
\end{abstract}

\section{Introduction}

Rolling bearing plays a very important role in the rotating system. It is one of the most commonly used and most easily damaged parts in rotating machinery, It is important to find out the early fault of bearing in time. However, the research on fault diagnosis of bearings at home and abroad mainly focuses on the research of fault diagnosis methods ${ }^{[1,2]}$, Therefore, in order to carry out the fault diagnosis of rolling bearing more effectively, it is necessary to study the vibration characteristics of rolling bearing. Kankar ${ }^{[3]}$ established the fault characterization model of rolling inner and outer ring by increasing the amount of clearance, and study the local fault modeling method based on displacement excitation. Dong Yabin ${ }^{[4]}$ etc established a rolling bearing model which considers the width, depth and load area. Xu Kejun ${ }^{[5]}$ etc Considered the influence of rolling through the inner ring fault release deformation and established a single rolling bearing inner ring pitting corrosion fault model, the defect model was improved. However, the bearing is only the supporting part of the rotor system, The vibration signal collected by the sensor is a set of vibration response of the whole rotor system, and the operating environment is more complex than the boundary condition of the bearing fault dynamics modeling.Therefore, it is of great significance to analysis the dynamic characteristics depth of the rolling bearing rotor system by putting the bearing in the rotor system for the study of the actual working conditions and the follow-up application of the bearing fault diagnosis.

\section{Dynamic model of dual rotor rolling bearing coupling system of engine}

Fig. 1 is a double rotor rolling bearing box coupling dynamic model. The two ends of the high pressure rotor and the low pressure rotor are respectively supported by 2 identical rolling bearings. In the coupled model, considering the rotor in initial forced vibration under unbalance, and because of the change of stiffness of bearing vibration of flexible $\mathrm{VC}$, in the coupling system, each component interaction, mutual coupling, considering the elastic support between the bearing outer ring and bearing seat and squeeze oil film damper and its excitation machine casing vibration. the mass of the casing is $m_{c}, m_{r p 1} 、 m_{r p 2}$ are the mass of high voltage rotor and low pressure rotor turntable respectively; $m_{b L 1} 、 m_{b R 1}$ are the mass of bearing housings at left and right ends; $m_{r L 1} 、 m_{r R 1}$ are the mass of the left and right ends of the high pressure rotor; $m_{r L 2} 、 m_{r R 2}$ are the mass of the left and right ends of the LP rotor respectively; $m_{w L 1} 、 m_{w R 1}$ are supporting bearing outer ring mass of left and right ends; $m_{w L 2} 、 m_{w R 2}$ are intermediate bearing outer ring mass of left and right ends; $k_{1} 、 c_{1} 、 c_{r b 1}$ are the stiffness of the high pressure shaft 、 the damping of the rotor at the disc the damping at both ends of bearing; $k_{2}$ 、 $c_{2} 、 c_{r b 2}$ are the stiffness of the low pressure shaft, the 
damping of the rotor at the disc 、 the damping at both ends of bearing; $k_{t R H 1} 、 k_{t R V 1}$ are transverse and vertical stiffness of elastic support between the right support bearing outer ring and right bearing bracket; $k_{t L H 1} 、 k_{t L V 1}$ are transverse and vertical stiffness of elastic support between the left support bearing outer ring and left bearing bracket; $k_{f R H 1}$ 、 $k_{f R V 1}$ are the lateral and vertical elastic support stiffness between the right bearing seat and the casing; $k_{f L H 1} 、 k_{f L V 1}$ are the lateral and vertical elastic support stiffness between the left bearing seat and the casing; $k_{c H} 、 k_{c V}$ were the transverse and vertical elastic support stiffness between the casing and the foundation; $c_{t L H 1}$ and $c_{t L V 1}$ were transverse and vertical bearing to squeeze film damping between the left support bearing outer ring and left bearing bracket; $c_{t R H 1}$ and $c_{t R V 1}$ were transverse and vertical bearing to squeeze film damping between the right support bearing outer ring and right bearing bracket; $c_{f R H 1}$ 、 $c_{f R V 1}, c_{f L H 1}$ and $c_{f L V 1}$ were the transverse and vertical squeeze film damping between the casing and the left bearing seat; $c_{f R H 1}$ and $c_{f R V 1}$ are the transverse and vertical squeeze film damping between the casing and the right bearing seat; $c_{c H} 、 c_{c V}$ are the transverse and vertical to squeeze film damping between the casing and the foundation; $e_{1} 、 e_{2}$ are mass eccentricity of high and low pressure rotor at the disk; $\omega_{1} 、 \omega_{2}$ are speed of high and low pressure rotor. $F_{x b L 1}$ and $F_{y b L 1}$ are respectively the $x$ and $y$ forces acting on the left end of the supporting bearing on the shaft, $F_{x b R 1}$ and $F_{y b R 1}$ are respectively the $x$ and $y$ forces acting on the right end of the supporting bearing. The differential equations of the system can be obtained by Newton's second law:

$$
\begin{aligned}
& m_{r p 1} \ddot{x}_{r p 1}+k_{1}\left(x_{r p 1}-x_{r R 1}\right)+k_{1}\left(x_{r p 1}-x_{r L 1}\right)+c_{1} \dot{x}_{r p 1}=m_{r p 1} e_{1} \omega_{1}^{2} \cos \omega_{1} t \\
& m_{r p 1} \ddot{y}_{r p 1}+k_{1}\left(y_{r p 1}-y_{r R 1}\right)+k_{1}\left(y_{r p 1}-y_{r L 1}\right)+c_{1} \dot{y}_{r p 1}=m_{r p 1} e_{1} \omega_{1}^{2} \sin \omega_{1} t-m_{r p 1} g \\
& m_{b R 1} \ddot{x}_{b R 1}+k_{f R H 1}\left(x_{b R 1}-x_{c}\right)+c_{f R H 1}\left(\dot{x}_{b R 1}-\dot{x}_{c}\right)+k_{t R H 1}\left(x_{b R 1}-x_{w R 1}\right)+c_{t R H 1}\left(\dot{x}_{b R 1}-\dot{x}_{w R 1}\right)=0 \\
& m_{b R 1} \ddot{y}_{b R 1}+k_{f R V 1}\left(y_{b R 1}-y_{c}\right)+c_{f R V 1}\left(\dot{y}_{b R 1}-\dot{y}_{c}\right)+k_{t R V 1}\left(y_{b R 1}-y_{w R 1}\right)+c_{t R V 1}\left(\dot{y}_{b R 1}-\dot{y}_{w R 1}\right)=-m_{b R 1} g \\
& m_{b L 1} \ddot{x}_{b L 1}+k_{f L H 1}\left(x_{b L 1}-x_{c}\right)+c_{f L H 1}\left(\dot{x}_{b L 1}-\dot{x}_{c}\right)+k_{t L H 1}\left(x_{b L 1}-x_{w L 1}\right)+c_{t L H 1}\left(\dot{x}_{b L 1}-\dot{x}_{w L 1}\right)=0 \\
& m_{b L 1} \ddot{y}_{b L 1}+k_{f L V 1}\left(y_{b L 1}-y_{c}\right)+c_{f L V 1}\left(\dot{y}_{b L 1}-\dot{y}_{c}\right)+k_{t L V 1}\left(y_{b L 1}-y_{w L 1}\right)+c_{t L V 1}\left(\dot{y}_{b L 1}-\dot{y}_{w R 1}\right)=-m_{b L 1} g \\
& \left(m_{r R 1}+m_{w R 2}\right) \ddot{x}_{r R 1}+k_{1}\left(x_{r R 1}-x_{r p 1}\right)+c_{r b 1} \dot{x}_{r R 1}-F_{x b R 1}+F_{x b R 2}=0 \\
& \left(m_{r R 1}+m_{w R 2}\right) \ddot{y}_{r R 1}+k_{1}\left(y_{r R 1}-y_{r p 1}\right)+c_{r b 1} \dot{y}_{r R 1}-F_{y b R 1}+F_{y b R 2}=-\left(m_{r R 1}+m_{w R 2}\right) g \\
& \left(m_{r L 1}+m_{w L 2}\right) \ddot{x}_{r L 1}+k_{1}\left(x_{r L 1}-x_{r p 1}\right)+c_{r b 1} \dot{x}_{r L 1}-F_{x b L 1}+F_{x b L 2}=0 \\
& \left(m_{r L 1}+m_{w L 2}\right) \ddot{y}_{r L 1}+k_{1}\left(y_{r L 1}-y_{r p 1}\right)+c_{r b 1} \dot{y}_{r L 1}-F_{y b L 1}+F_{y b L 2}=-\left(m_{r L 1}+m_{w L 2}\right) g \\
& \left\{m_{w R 1} \ddot{x}_{w R 1}+k_{t R H 1}\left(x_{w R 1}-x_{b R 1}\right)+c_{t R H 1}\left(\dot{x}_{w R 1}-\dot{x}_{b R 1}\right)+F_{x b R 1}=0\right. \\
& m_{w R 1} \ddot{y}_{w R 1}+k_{t R V 1}\left(y_{w R 1}-y_{b R 1}\right)+c_{t R V 1}\left(\dot{y}_{w R 1}-\dot{y}_{b R 1}\right)+F_{y b R 1}=-m_{w R 1} g \\
& m_{w L 1} \ddot{x}_{w L 1}+k_{t L H 1}\left(x_{w L 1}-x_{b L 1}\right)+c_{t L H 1}\left(\dot{x}_{w L 1}-\dot{x}_{b L 1}\right)+F_{x b L 1}=0 \\
& m_{w L 1} \ddot{y}_{w L 1}+k_{t L V 1}\left(y_{w L 1}-y_{b L 1}\right)+c_{t L V 1}\left(\dot{y}_{w R 1}-\dot{y}_{b L 1}\right)+F_{y b L 1}=-m_{w L 1} g \\
& m_{r p 2} \ddot{x}_{r p 2}+k_{2}\left(x_{r p 2}-x_{r R 2}\right)+k_{2}\left(x_{r p 2}-x_{r L 2}\right)+c_{2} \dot{x}_{r p 2}=m_{r p 2} e_{2} \omega_{2}{ }^{2} \cos \omega_{2} t \\
& m_{r p 2} \ddot{y}_{r p 2}+k_{2}\left(y_{r p 2}-y_{r R 2}\right)+k_{2}\left(y_{r p 2}-y_{r L 2}\right)+c_{2} \dot{y}_{r p 2}=m_{r p 2} e_{2} \omega_{2}^{2} \sin \omega_{2} t-m_{r p 2} g \\
& m_{r R 2} \ddot{x}_{r R 2}+k_{2}\left(x_{r R 2}-x_{r p 2}\right)+c_{r b 2} \dot{x}_{r R 2}-F_{x b R 2}=0 \\
& m_{r R 2} \ddot{y}_{r R 2}+k_{2}\left(y_{r R 2}-y_{r p 2}\right)+c_{r b 2} \dot{y}_{r R 2}-F_{y b R 2}=-m_{r R 2} g \\
& m_{r L 2} \ddot{x}_{r L 2}+k_{2}\left(x_{r L 2}-x_{r p 2}\right)+c_{r b 2} \dot{x}_{r L 2}-F_{x b L 2}=0 \\
& m_{r L 2} \ddot{y}_{r L 2}+k_{2}\left(y_{r L 2}-y_{r p 2}\right)+c_{r b 2} \dot{y}_{r L 2}-F_{y b L 2}=-m_{r L 2} g \\
& m_{c} \ddot{x}_{c}+k_{c H} x_{c}+c_{c H} \dot{x}_{c}+k_{f R H 1}\left(x_{c}-x_{b R 1}\right)+k_{f L H 1}\left(x_{c}-x_{b L 1}\right)+c_{f R H 1}\left(\dot{x}_{c}-\dot{x}_{b R 1}\right)+c_{f L H 1}\left(\dot{x}_{c}-\dot{x}_{b L 1}\right)=0 \\
& m_{c} \ddot{y}_{c}+k_{c V} y_{c}+c_{c V} \dot{y}_{c}+k_{f R H 1}\left(y_{c}-y_{b R 1}\right)+k_{f L H 1}\left(y_{c}-y_{b L 1}\right)+c_{f R H 1}\left(\dot{y}_{c}-\dot{y}_{b R 1}\right)+c_{f L H 1}\left(\dot{y}_{c}-\dot{y}_{b L 1}\right)=-m_{c} g
\end{aligned}
$$




\section{Rolling bearing force model}

The rolling bearing is composed of an inner ring, an outer ring, a rolling body and a retainer. In this paper, the bearing outer ring is fixed on the bearing seat, and the inner ring is fixed on the rotating shaft, as shown in Fig.2.

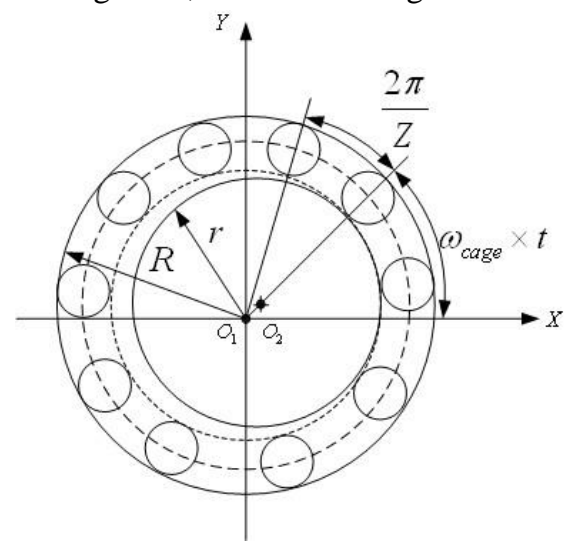

Fig.2.Ball bearing model

The ball in the bearing is arranged between the inner and the outer raceway, and the ball is pure rolling. Reference[6], the rolling bearing can be obtained as:

$$
\left\{\begin{array}{c}
F_{x}=\sum_{j=1}^{Z} C_{b}\left(x \cos \theta_{j}+y \sin \theta_{j}-\delta_{0}-\delta_{D}\right)^{\frac{3}{2}} \bullet \\
H\left(x \cos \theta_{j}+y \sin \theta_{j}-\delta_{0}-\delta_{D}\right) \cos \theta_{j} \\
F_{y}=\sum_{j=1}^{Z} C_{b}\left(x \cos \theta_{j}+y \sin \theta_{j}-\delta_{0}-\delta_{D}\right)^{\frac{3}{2}} \bullet \\
H\left(x \cos \theta_{j}+y \sin \theta_{j}-\delta_{0}-\delta_{D}\right) \sin \theta_{j}
\end{array}\right.
$$

The $C_{b}$ is Hertz contact stiffness that is related to the material and shape of the contact, $H(\bullet)$ is the heaviside function, When the function variable is greater than 0 , the function value is 1 Otherwise it is $0 . \delta_{0}$ is initial clearance for rolling bearing, $\delta_{D}$ is the gap caused by local damage of bearing, $\theta_{j}$ is the angular position of the Jth ball. $\theta_{j}=\omega_{\text {Cage }} \times t+\frac{2 \pi}{Z}(j-1), \quad(j=1,2, \cdots, Z) . \omega_{\text {Cage }}$ is cage rotation speed。Assuming the radius of outer raceway is $R$, radius of inner raceway is $r$, there is $\omega_{\text {Cage }}=\frac{\omega \times r}{R+r}, \mathrm{VC}$ is the frequency of ball passing, so, $Z=\omega \times\left(\frac{r}{R+r} \times Z\right)=\omega \times B_{N}, B_{N}$ is the ratio of $\mathrm{VC}$ frequency to rotation frequency, $B_{N}=\frac{r}{R+r} \times Z, Z$ is the number of balls 。
Thus, the bearing force produced by the bearing can be obtained in the double rotor dynamic modelis :

1) when $x=x_{r R 1}-x_{w R 1}, y=y_{r R 1}-y_{w R 1}, \omega=\omega_{1}$; then $F_{x b R 1}=F_{x}, \quad F_{y b R 1}=F_{y}$

2) when $x=x_{r L 1}-x_{w L 1}, y=y_{r L 1}-y_{w L 1}, \omega=\omega_{1}$; then $F_{x b L 1}=F_{x}, \quad F_{y b L 1}=F_{y}$

The bearing force produced by the intermediate bearing is (assuming that the high and low pressure rotors rotate in the same direction and $\omega_{1}>\omega_{2}$ )

1) when $x=x_{r R 2}-x_{r R 1}, y=y_{r R 2}-y_{r R 1}, \quad \omega=\omega_{1}-\omega_{2}$; then $F_{x b R 2}=F_{x}, \quad F_{y b R 2}=F_{y}$

2) when $x=x_{r L 2}-x_{r L 1}, \quad y=y_{r L 2}-y_{r L 1}, \quad \omega=\omega_{1}-\omega_{2}$; then $F_{x b L 2}=F_{x}, \quad F_{y b L 2}=F_{y}$

\section{The fault of rolling bearing inner ring}

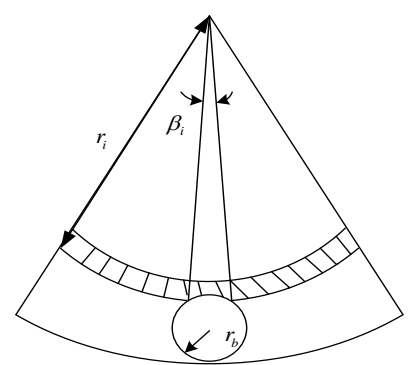

(a) Schematic diagram of inner fault

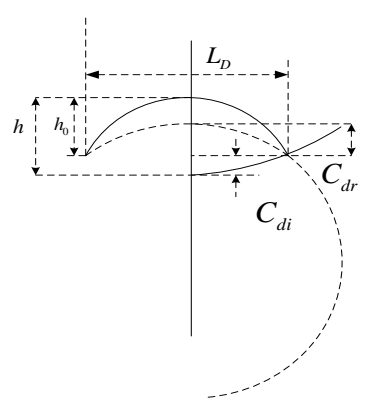

(b) Fault enlargement
Fig.3.Fault bearing inner ring

As can be seen from Fig.3, Defect depth is usually defined as $h_{0}$, However, due to the fact that the inner ring can not be a complete rigid body in actual contact, it will also produce deformation, Due to ignoring the deformation of the inner ring, $h_{0}$ can not fully represent the severity of the inner ring fault. Depth should be $h_{0}$ and the inner ring shaped variable $C_{d i}$ addition, that is $h$ shown in the diagram. It is assumed that the gap is $\delta_{D}$, When the rolling body through the inner defect and contact with the bottom of the defect, the maximum release is $\delta_{D \max }$ equals to $h$. When the rolling body passes through the inner defect but does not touch the bottom of the defect, the maximum deformation is $\delta_{D \max }=C_{d r}+C_{d i}$. Can be obtained by schematic diagram:

$$
C_{d r}=r_{b}-\sqrt{r_{b}^{2}-\left(L_{D} / 2\right)^{2}}
$$




$$
C_{d i}=r_{i}-\sqrt{r_{i}^{2}-\left(L_{D} / 2\right)^{2}} .
$$

The maximum number of variables that roll out through the outer ring:

$$
\delta_{D \max }= \begin{cases}h & h<\delta_{D \max } \\ \delta_{D \max } & h \geq \delta_{D \max }\end{cases}
$$

Next, to determine the condition of bearing clearance change caused by damage, Set the position angle of the jth ball is $\theta_{j}, \theta_{j}=\omega_{\text {Cage }} \times t+2 \pi(j-1) / Z$.The location of the fault center at the initial time is $\varphi_{i n}$, The angle position of the $\mathrm{t}$ moment defect is located in $\theta_{i n}, \theta_{i n}=\omega_{1} \times t+\varphi_{\text {in }}$, Defect angle size is $\beta_{i}$, and $\beta_{i}=2 \arcsin \left(L_{D} / D_{i n}\right), \quad D_{i n}$ is outer diameter。Because only in the damaged area (namely the ball rolling body position in the center of the fault angle) occurs, therefore, in the rolling body rotation range, bearing clearance change conditions can be expressed as: $\theta_{i n}-\beta_{i} / 2 \leq \theta_{j} \leq \theta_{i n}+\beta_{i} / 2$ then:

$$
\delta_{D}=\left\{\begin{array}{l}
\delta_{D \max } \\
\left(0<\left|\left[\theta_{j}-\theta_{i n}\right] \bmod (2 \pi)\right|<\beta_{i} / 2\right) . \\
0
\end{array}\right.
$$

In the calculation of dynamics, put the change of bearing clearance can be into the formula (2).

\section{Simulation analysis of rolling bearing fault}

\subsection{Calculation parameters}

In this paper, with reference to the actual aero-engine dual rotor model ${ }^{[7]}$, the initial parameters of the double rotor system are selected as follows:

$$
\begin{aligned}
& m_{r p 1}=14.06 \mathrm{~kg}, m_{r p 2}=20.22 \mathrm{~kg}, k_{1}=1.0 \times 10^{7} \mathrm{~N} / \mathrm{m}, \\
& c_{1}=2100 \mathrm{~N} \bullet \mathrm{s} / \mathrm{m}, c_{r b 1}=1050 \mathrm{~N} \bullet \mathrm{s} / \mathrm{m}, k_{2}=2.0 \times 10^{7} \mathrm{~N} / \mathrm{m}, \\
& c_{1}=2200 \mathrm{~N} \bullet \mathrm{s} / \mathrm{m}, c_{r b 2}=1050 \mathrm{~N} \bullet \mathrm{s} / \mathrm{m}, m_{b L 1}=m_{b R 1}=9 \mathrm{~kg}, \\
& m_{b L 2}=m_{b R 2}=13 \mathrm{~kg}, e_{1}=e_{2}=5 \times 10^{-5} \mathrm{~m}, \\
& c_{f L H 1}=c_{f L V 1}=c_{f R H 1}=c_{f R V 1}=2100 \mathrm{~N} \bullet \mathrm{s} / \mathrm{m}, \\
& k_{t L H 1}=k_{t L V 1}=k_{t R H 1}=k_{t R V 1}=2.5 \times 10^{7} \mathrm{~N} / \mathrm{m}, \\
& k_{f L H 1}=k_{f L V 1}=k_{f R H 1}=k_{f R V 1}=2.5 \times 10^{7} \mathrm{~N} / \mathrm{m}, \\
& m_{r L 1}=m_{r R 1}=2.0 \mathrm{~kg}, c_{t L H 1}=c_{t L V 1}=c_{t R H 1}=c_{t R V 1}=1050 \mathrm{~N} \bullet \mathrm{s} / \mathrm{m}, \\
& k_{c H}=k_{c V}=2.5 \times 10^{9} \mathrm{~N} / \mathrm{m}, c_{c H}=2100 \mathrm{~N} \bullet \mathrm{s} / \mathrm{m}, m_{r L 2}=m_{r R 2}=2.0 \mathrm{~kg}, \\
& m_{w L 1}=m_{w R 1}=2.0 \mathrm{~kg}, m_{c}=50 \mathrm{~kg},
\end{aligned}
$$

\begin{tabular}{|c|c|c|c|c|c|c|}
\hline Bearing & $\begin{array}{l}\text { Outer } \\
\text { radius }\end{array}$ & $\begin{array}{l}\text { Inner } \\
\text { radius }\end{array}$ & $\begin{array}{l}\text { Number } \\
\text { of ball }\end{array}$ & $\begin{array}{l}\text { Contact } \\
\text { stiffness }\end{array}$ & $\begin{array}{l}\text { Bearing } \\
\text { clearance }\end{array}$ & DN \\
\hline name & $\mathrm{R} / \mathrm{mm}$ & $\mathrm{r} / \mathrm{mm}$ & $Z$ & $C_{b} / 10^{9}\left(\mathrm{~N} / \mathrm{m}^{3 / 2}\right)$ & $r_{0} / \mu m$ & \\
\hline JIS6306 & 63.9 & 40.1 & 8 & 13.34 & 5 & 3.08 \\
\hline SKF6002 & 14.13 & 9.37 & 9 & 7.055 & 2 & 3.6 \\
\hline
\end{tabular}

Select JIS6306 and SKF6002 rolling bearings in literature ${ }^{[7]}$. As the support bearing system and intermediary bearing, its parameters are shown in Table I:

Table.I Bearing parameters

\subsection{Inner fault response}

Inner damage width $L_{D}=0.3 \mathrm{~mm}$, depth of damage $h_{0}=2.5 \mathrm{~mm}$, rotor speed is $30 \mathrm{r} / \mathrm{s}$, and $f_{r}=30 \mathrm{~Hz}$, The characteristic frequency of inner ring damage can be calculated: $f_{\text {in }}=4.9154 \times f_{r}=4.9154 \times 30 \cong 148 \mathrm{~Hz}$, Inner fault period $T_{i n}=1 / f_{\text {in }} \cong 0.0068$ s. The numerical simulation of the bearing failure equation of the system is carried out, and the simulation results of the inner fault bearing fault are shown.
As can be seen from the (b), when there is a defect in the inner ring, the bearing seat will have a significant impact response, the vibration speed will suddenly increase, after the impact, the system will produce a series of attenuation vibration, The impact period is $0.033 \mathrm{~s}$. (c) for its full spectrum, we can see that in the vicinity of the frequency of two $2000 \mathrm{~Hz}$ resonance peak, the edge of the band spacing of $148 \mathrm{~Hz}$ (about 1/0.0068), (d) for the amplification of high frequency spectrum, 1887, 2035 of the difference in the figure is exactly the value of the internal frequency $f_{\text {in }}$, that is the characteristic frequency of single point damage. In addition, due to the inner fault position continuously with bearing rotation and change, so the inner fault impact will be modulated by the rotational frequency, for the existence of 
interval is equal to the frequency in the inner ring around the axis $(30 \mathrm{~Hz})$ of the line, such as $1857 \mathrm{HZ}, 1887 \mathrm{HZ}$. The ${ }^{[8]}$ pointed out that There is a gradual decrease in the frequency spectrum of the inner ring damage at each order frequency of the inner fault frequency and centered on the order frequency,on both sides of the line with a frequency of rotation. Therefore, the results of this paper are in agreement with the conclusion, which verifies the correctness of the model.

\section{Conclusion}

according to the actual dual rotor system of aero-engine, a two rotor bearing bearing shell coupling system is established. In consideration of the bearing clearance, nonlinear Hertzian contact force and the vibration of $\mathrm{VC}$ at the same time, considering the casing movement, elastic support and squeeze film damper. The numerical integration method is used to simulate and verify.

the damage dynamic model of bearing inner ring on the basis of the model of the coupled system. In the research of the bearing fault, the vibration of the rotor system and the casing is fully taken into account, which is more in line with the objective reality. The "Local first and then whole" ideas on the fault model for dynamic simulation, through calculation and analysis to verify the correctness of the rolling bearing damage dynamics model proposed in this paper and the dual rotor ball bearing stator coupling system machine.

\section{Acknowledgment}

This work is supported by National Natural Science Foundation of China (No.51505486), all support is gratefully acknowledged.

\section{References}

[1] Sun W Yang G, Chen Q, et al. Fault diagnosis of rolling bearing based on wavelet transform and envelope spectrum correlation[J]. Journal of Vibration and Control, 2013, 19(6):924-941.

[2] CAI Yan-ping, LIAI-hua, et al. Roller. bearing fault detection using improved envelope spectrum analysis based on EMD and spectrum kurtosis [J]. Journal of Vibration and shock,2011, 30(2):167-191.

[3] Kankar P K, Sharma S, Harsha S. Fault Diagnosis of High Speed Rolling Element Bearings Due to Localized Defects Using Response Surface Method[J]. Transactions of the ASME, Journal of Dynamic Systems, Measurement, and Control, 2011, 13:1-14.

[4] DONG Ya-bin, LIAO Ming-fu, GAO Qi. Dynamics analysis on rolling element bearings with localized defects [J]. Heavy Machinery, 2012(3): 148-152.

[5] XU Ke-jun, REN shuai, et al. Dynamics Model Establishment and Simulation of Rolling Bearing Element with Inner Race Defect [J]. Aerogine 2015,41(4): 8-11.
[6] CHEN Guo. Dynamic analysis of ball bearing faults in rotor-ball bearing-stator coupling system [J]. Journal of Vibration Engineering. 2008, 21(6):577-587.

[7] Aircraft engine design manual, editorial board. Aero engine design manual (Nineteenth volume), rotor dynamics and whole machine vibration $[\mathrm{M}]$ : Aviation Industry Press, 2000: 43-44.

[8] MEI Hong-bin. Vibration monitoring and diagnosis of rolling bearing [M]. Beijing: China Machine Press, 1996. 


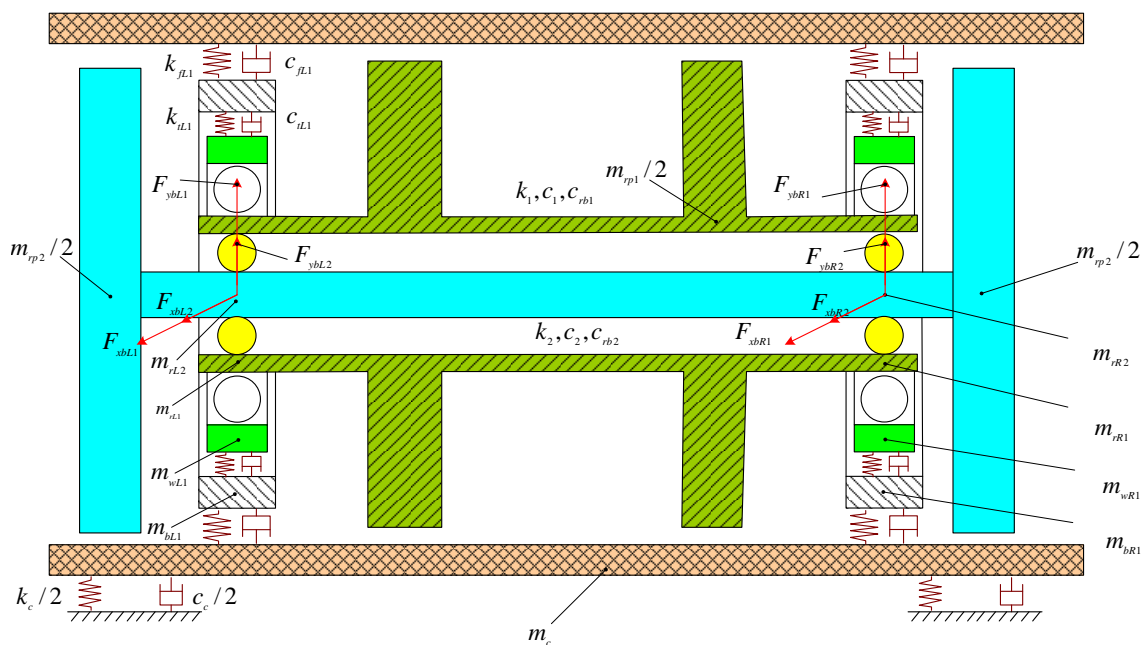

Fig.1.The whole model for aero-engine

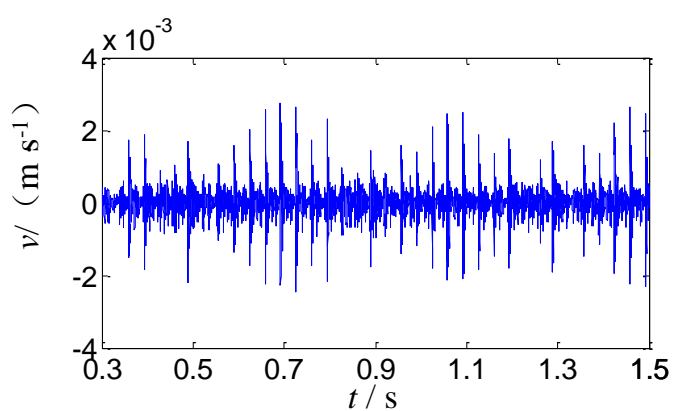

(a) Time waveform

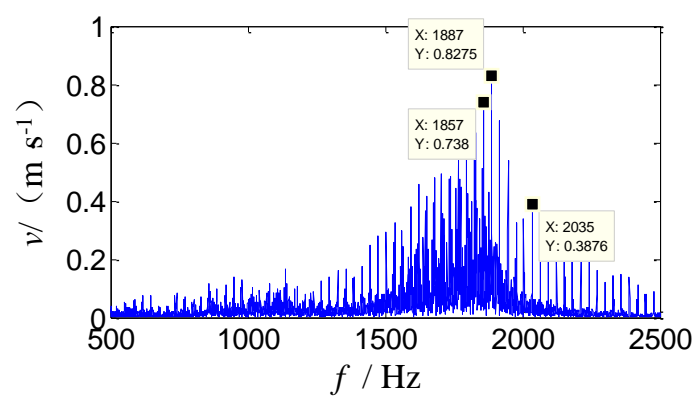

(c) spectrum

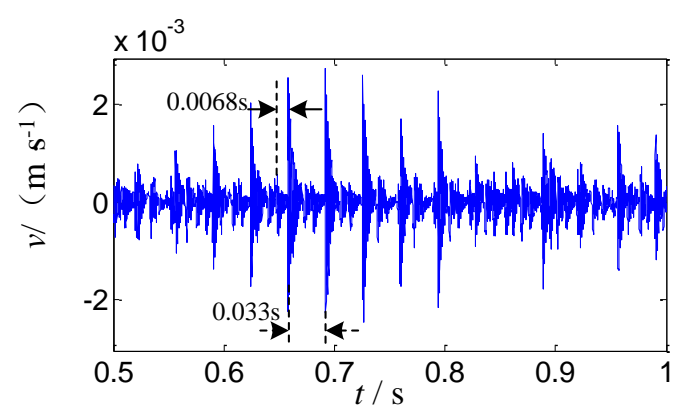

(b) Time waveform

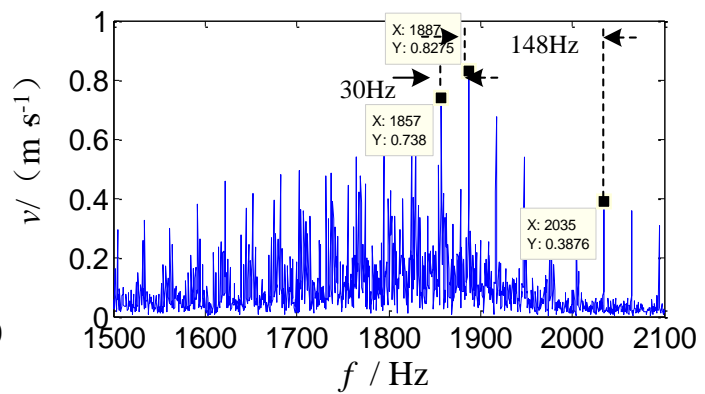

(d) Resonance region enlargement

Fig.4. Inner ring fault response 